\title{
Laparoscopic Intersphincteric Resection: A Feasible Technique or the Treatment of Choice for Patients with Low Rectal Cancer?
}

\author{
K.C.M.J. Peeters L.P.S. Stassen \\ Department of Surgery, Maastricht University Medical Centre, Maastricht, The Netherlands
}

For many years rectal cancer has posed a challenge to oncologists, with locoregional recurrences of up to $30 \%$ being an important cause of debilitating death in the past. Since Miles introduced his abdominoperineal resection more than 100 years ago, techniques have been refined substantially, inflicting less trauma on the patient. Stapling techniques have made very distal anastomoses possible and meticulous dissection in the right anatomical planes has reduced the necessity of a wide distal resection margin. As such, the number of patients undergoing sphincter-saving surgery has increased. Transanal endoscopic microsurgery for early rectal cancers and the introduction of laparoscopy have further contributed in this respect. Apart from these major surgical advances, novel (neo)adjuvant treatment regimens have further increased both local control and survival. Preoperative radiotherapy has reduced the likelihood of local failure, and chemoradiotherapy may even induce a complete clinical response. Although this occurs only in a minority of patients, the impact is significant: wait-and-see protocols omitting surgery are currently being explored in several parts of the world.

Parallel to these advances in rectal cancer treatment, more attention is being paid to the impact of several treatment options on quality of life $(\mathrm{QoL})$. In general, a stoma is considered to have a negative impact on QoL. Nevertheless, major improvements have been made in stoma care and surgeons are increasingly aware of the necessity of creating a stoma that has optimal function. Patients with a permanent stoma do not necessarily end up with an unacceptable functional result. They might even have a better QoL than patients with colorectal or coloanal anastomoses as the latter patient group often suffers from fecal incontinence due to the lack of filling capacity of the neorectum [1]. Not only medical considerations but also the cultural mindset in the heads of both patients and their doctors determine whether a stoma is constructed or not.

Yamamoto et al. [2] describe in a single-center report their results of laparoscopic intersphincteric resection (ISR) for patients with distal rectal cancer. Only 29 patients were described in a study period of almost 10 years, suggesting a certain selection bias. Of these, 7 patients were excluded as matched cases could not be identified in the control group. ISR in rectal cancer is based on several facts and assumptions: through meticulous dissection it has become a feasible technique, it is safe in certain oncological stages, and it results in a superior QoL for the patients. However, the exact place of this technique, performed open or laparoscopically, is not completely clear.

The surgeon's expertise and the will of both surgeons and patients to preserve the anus certainly influences the likelihood that this technique will be used. As the authors describe, in order to make it a safe procedure a skilled surgical team and adequate postoperative care are prerequisites. With acceptable morbidity and absent mortality, the Japanese colleagues seem to have fulfilled this demand. Of course, the oncological outcome after (laparoscopic) ISR is of key importance. When a safe distal margin of 1-2 cm can be achieved most authors consider

\section{KARGER}

Fax +4161306 1234 E-Mail karger@karger.ch www.karger.com

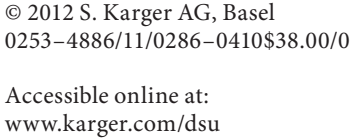

K.C.M.J. Peeters

Department of Surgery, Maastricht University Medical Centre

P. Debyelaan 25, PO Box 5800

NL-6202 AZ Maastricht (The Netherlands)

E-Mail koen.peeters@mumc.nl 
ISR feasible [3-5]. Local invasion of the internal sphincter is acceptable, whereas invasion of the external sphincter is not. This implies a complete preoperative workup to select patients that are candidates for ISR. In the current report, details on pathological examination are scarce: 'positive margin was 0 '. It is well known that thorough and adequate examination of the resection margins by the pathologist is pivotal: involvement of the circumferential plane of resection is the most important predictor for local failure [6], and the distal margin is at risk in patients with distal rectal cancer, especially in those who undergo sphincter-preserving surgery. Despite negative margins in the present report, 2 patients developed a local recurrence (6.9\%). Of course, this seems an acceptable figure, but the majority of the patients had a stage I tumor, making a local recurrence hard to endure.

In the present study, functional results are hardly reported. On the one hand, this is not surprising as the authors state in the title of their manuscript that they report on short-term outcomes only. On the other hand, data on late functional outcome in patients treated as early as 2002 could have been of interest as well. However, this paper is no exception as the reports on continence vary considerably. In some studies no information is given other than 'good preoperative continence and good sphincter function' $[4,7,8]$, while in others a combination of thorough clinical examination with adequate scoring and anorectal physiological testing is performed $[9,10]$. Despite this bias in underreporting, ISR is known to have its downsides: a clear reduction of anal resting pressure is observed (29\%) [3], with urgency in $19-59 \%$ of patients and daytime and nocturnal leakage in 15 and 20\%, respectively [11]. Other series report even higher percentages, whereas colonic J-pouch reconstruction significantly improves the functional outcome $[12,13]$.

In conclusion, (laparoscopic) ISR is a treatment option for rectal cancer patients provided surgical expertise is available, making it a safe procedure. A detailed preoperative workup is needed to rule out external sphincter invasion and to determine the need for preoperative (chemo)radiotherapy. Finally, careful assessment of the patient's continence prior to rectal cancer treatment is important as well as his/her anticipated QoL after treatment. Weighing these arguments, it should be decided whether (laparoscopic) ISR is the preferred technique in patients with rectal cancer. These efforts seem worthwhile as a growing majority of rectal cancer patients will overcome their disease and therefore have to deal with the sequelae of rectal cancer treatment for the rest of their lives.

\section{References}

$>1$ Marijnen CA, van de Velde CJ, Putter H, van den Brink M, Maas CP, Martijn H, Rutten HJ, Wiggers T, Kranenbarg EK, Leer JW, Stiggelbout AM: Impact of short-term preoperative radiotherapy on health-related quality of life and sexual functioning in primary rectal cancer: report of a multicenter randomized trial. J Clin Oncol 2005;23: 1847-1858.

$\checkmark 2$ Yamamoto S, et al: Short-term outcomes of laparoscopic intersphincteric resection for lower rectal cancer and comparison with open approach. Dig Surg 2011;28:404-409.

3 Schiessel R, Karner-Hanusch J, Herbst F, Teleky B, Wunderlich M: Intersphincteric resection for low rectal tumours. Br J Surg 1994;81:1376-1378.

4 Saito N, Ono M, Sugito M, Ito M, Morihiro M, Kosugi C, Sato K, Kotaka M, Nomura S, Arai M, Kobatake T: Early results of intersphincteric resection for patients with very low rectal cancer: an active approach to avoid a permanent colostomy. Dis Colon Rectum 2004;47:459-466.
5 Rullier E, Goffre B, Bonnel C, Zerbib F, Caudry M, Saric J: Preoperative radiochemotherapy and sphincter-saving resection for $\mathrm{T} 3$ carcinomas of the lower third of the rectum. Ann Surg 2001;234:633-640.

6 Nagtegaal ID, Marijnen CA, Kranenbarg EK, van de Velde CJ, van Krieken JH: Circumferential margin involvement is still an important predictor of local recurrence in rectal carcinoma: not one millimeter but two millimeters is the limit. Am J Surg Pathol 2002;26:350-357.

7 Kohler A, Athanasiadis S, Ommer A, Psarakis E: Long-term results of low anterior resection with intersphincteric anastomosis in carcinoma of the lower one-third of the rectum: analysis of 31 patients. Dis Colon Rectum 2000;43:843-850.

$\checkmark 8$ Hohenberger W, Merkel S, Matzel K, Bittorf B, Papadopoulos T, Gohl J: The influence of abdomino-peranal (intersphincteric) resection of lower third rectal carcinoma on the rates of sphincter preservation and locoregional recurrence. Colorectal Dis 2006;8:2333.
$>$ Renner K, Rosen HR, Novi G, Holbling N, Schiessel R: Quality of life after surgery for rectal cancer: do we still need a permanent colostomy? Dis Colon Rectum 1999;42: 1160-1167.

10 Schiessel R, Novi G, Holzer B, Rosen HR, Renner K, Holbling N, Feil W, Urban M: Technique and long-term results of intersphincteric resection for low rectal cancer. Dis Colon Rectum 2005;48(10):1858-1865, discussion 1865-1867.

11 Braun J, Treutner KH, Winkeltau G, Heidenreich U, Lerch MM, Schumpelick V: Results of intersphincteric resection of the rectum with direct coloanal anastomosis for rectal carcinoma. Am J Surg 1992;163:407-412.

$\checkmark 12$ Bittorf B, Stadelmaier U, Gohl J, Hohenberger W, Matzel KE: Functional outcome after intersphincteric resection of the rectum with coloanal anastomosis in low rectal cancer. Eur J Surg Oncol 2004;30:260-265.

13 Park JG, Lee MR, Lim SB, Hong CW, Yoon SN, Kang SB, Heo SC, Jeong SY, Park KJ: Colonic J-pouch anal anastomosis after ultralow anterior resection with upper sphincter excision for low-lying rectal cancer. World J Gastroenterol 2005;11:2570-2573. 